Article

\title{
Application of Concentrated Growth Factors Membrane for Human Umbilical Cord Wharton's Jelly Mesenchymal Stem Cell Differentiation towards Keratinocytes
}

\author{
Chao-Hsing Kao
}

Citation: Kao, C.-H. Application of Concentrated Growth Factors Membrane for Human Umbilical Cord Wharton's Jelly Mesenchymal Stem Cell Differentiation towards Keratinocytes. Separations 2021, 8, 61. https://doi.org/10.3390/ separations 8050061

Academic Editor: Francisco J. Barba

Received: 22 February 2021

Accepted: 28 April 2021

Published: 7 May 2021

Publisher's Note: MDPI stays neutral with regard to jurisdictional claims in published maps and institutional affiliations.

Copyright: (C) 2021 by the author. Licensee MDPI, Basel, Switzerland. This article is an open access article distributed under the terms and conditions of the Creative Commons Attribution (CC BY) license (https:// creativecommons.org/licenses/by/ $4.0 /)$.
Kao Chao-Hsing Dermatologic Clinic, No. 277, Jiuru 2nd Rd., Sanmin Dist., Kaohsiung City 807, Taiwan; kaochclinic@gmail.com; Tel.: +886-7-3220664

\begin{abstract}
Concentrated growth factors are extracted from platelet-rich plasma obtained from healthy adult veins by physical gradient centrifugation, and the activated platelets release various growth factors and cytokines, which can be further converted into concentrated growth factors liquid or gel preparations by different centrifuge tubes. These preparations are widely used in clinical treatments in various fields, such as dentistry, dermatology and surgery. In this article, concentrated growth factors gel and platelet-poor plasma gel obtained from six healthy adults were pressed into a concentrated growth factors membrane and platelet-poor plasma membrane. We examined whether the 3D fibrin mesh and the various concentrated growth factors within the concentrated growth factors membrane could be used as a bioscaffold for the human Wharton's jelly umbilical cord stem cell line or the $\mathrm{HaCaT}$ cell line to attach, proliferate and form epidermal-like tissue. We also aimed to implant umbilical cord stem cells on the concentrated growth factors membrane or platelet-poor plasma membrane, and further compare the characteristics of similar tissues after 4 weeks in in vitro culture. The results showed that human Wharton's jelly umbilical cord mesenchymal stem cells, implanted on the upper surface of the concentrated growth factors membrane, showed subsequent cell attachment and proliferation. After 4 weeks of ex vivo tissue culture, a multi-layer epidermal-like tissue formed on the upper surface of the membrane containing concentrated growth factors. This tissue had a minimum thickness of $89.91 \mu \mathrm{m}$ to a maximum of $204.19 \mu \mathrm{m}$, mean $\pm \mathrm{SD}=144.36 \mu \mathrm{m} \pm 43.14 \mu \mathrm{m}$. Sections of these multi-layer epidermal-like tissues were used for immunohistochemical staining. We found that $79.8 \% \pm 7.2 \%$ of the cells expressed the pancytokeratin marker, $29.5 \% \pm 9.4 \%$ of the cells expressed the P63 marker, and $71.7 \% \pm 3.9 \%$ of the cells expressed the vimentin marker. After the same 4 weeks in the in vitro culture, the HaCaT cells could attach to the concentrated growth factors membrane and proliferate to form a multi-layer tissue, The tissue had a minimum thickness of $63.17 \mu \mathrm{m}$ to a maximum of $100.26 \mu \mathrm{m}$, mean $\pm \mathrm{SD}=74.05 \mu \mathrm{m} \pm 13.44 \mu \mathrm{m}$. We found that $88.1 \% \pm 4.9 \%$ of the cells expressed the pancytokeratin marker, $63.6 \% \pm 11.4 \%$ of the cells expressed the P63 marker, and 79\% $\pm 9.9 \%$ of the cells expressed the vimentin marker. Also, after 4 weeks in the in vitro culture, it showed that umbilical cord stem cells could attach to the plateletpoor plasma membrane, proliferate and distribute in the whole-tissue sections. We found that $9.7 \% \pm 2.4 \%$ of the cells expressed the pancytokeratin marker, $7.45 \% \pm 1.9 \%$ of the cells expressed the P63 maker, and $95.9 \% \pm 3.7 \%$ of the cells expressed the vimentin marker. In terms of the percentage of umbilical cord stem cells expressing pancytokeratin, P63, or vimentin cell markers, there was a significant difference between cultivating in the concentrated growth factors membrane scaffold and the platelet-poor plasma membrane scaffolds. In terms of the percentage of umbilical cord stem cells or HaCaT cells (cultivating in the concentrated growth factors membrane) expressing pancytokeratin, P63, or vimentin cell markers, there was no significant difference. These results suggested that umbilical cord Wharton's jelly mesenchymal stem cells can use the concentrated growth factors membrane (composed of 3D fibrin mesh, and various growth factors and cytokines) as an effective and self-contained bioscaffold to differentiate towards keratinocytes-like cells. In the future, donors' own concentrated growth factors membrane can be applied as an auxiliary tool for autologous tissue regeneration.
\end{abstract}


Keywords: concentrated growth factors membrane; human umbilical cord Wharton's jelly mesenchymal stem cells; bioscaffold; keratinocyte stem cells

\section{Introduction}

Concentrated growth factors (CGF) are obtained from activated platelet-rich plasma by physical gradient centrifugation [1,2], and the activated platelets release various growth factors and cytokines [3-6]. They can be further converted into liquid preparations of concentrated growth factors or concentrated growth factors gel by different centrifugation approaches. The concentrated growth factors gel is rich in white blood cells contained in the bottom layer close to the clot of red blood cells. The advantage of using concentrated growth factors in clinical practice is that they can avoid the need to add artificial thrombin and anticoagulant in autologous use, but are still as effective as physically activated platelet-rich plasma obtained by gradient centrifugation. They are widely used in clinical treatment and research in dentistry $[3,7,8]$, dermatology $[9,10]$, plastic surgery $[11,12]$ and orthopedics [13]. Plasma fibrin is also a versatile scaffold in tissue engineering [14,15].

Human Wharton's jelly umbilical cord mesenchymal stem cells are multifunctional stem cells that can be transformed into fibroblasts, keratinocytes and various other cells under appropriate conditions [16-18].

In this paper, we in vitro cultured human Wharton's jelly umbilical cord stem cells with 3D fibrin mesh and concentrated growth factors in a membrane obtained from pressed concentrated growth factors gel. We then examined whether the concentrated growth factors membrane could be used as a good bioscaffold for the attachment, proliferation and transformation of human Wharton's jelly umbilical cord stem cells into epidermallike tissue.

\section{Materials and Methods}

\subsection{Preparation of Concentrated Growth Factors Membrane}

Approved by the IRB of Yuan's General Hospital (no. 20200602B), blood samples for in vitro tissue culture experiments were collected from six healthy adults ( 3 males and 3 females), and all experiments were repeated using the same procedure.

As previously reported $[6,10,11]$, to prepare CGF gel, we extracted $7 \mathrm{~mL}$ of venous blood from the human body into a sterile vacuum blood collection tube (BD Vacutainer, REF 367820, Becton Dickson and Company, Franklin Lakes, NJ, USA) without any anticoagulant; a silica coating was used on the inner tube wall. Then, we immediately placed the blood collection tube into the centrifuge and performed fractional gradient centrifugation (Medifuge CGF MF200100 Silfradent SRL, Sofin, FC, Italy) using the CGF protocol. Blood was divided into the following three layers after centrifugation: the upper, lower, and middle layers comprising of platelet-deprived plasma, red blood cells and fibrin gel, respectively (Figure 1A). The red blood cell clot beneath the fibrin gel was scraped off with scissors, after which three-fifths of the fibrin gel from the top was portioned off as platelet-poor plasma (PPP) gel, and the remaining two-fifths of the fibrin gel layer was portioned off as CGF gel (containing platelets, leukocytes, growth factors, CD34 + stem cells and some adhered red blood cells) (Figure 1B). The upper half of the CGF gel was devoid of white and red blood cells. After clamping the upper half of the CGF gel or upper half of PPP gel into the membrane with a mesh clip (Figure 1C,D), the fresh membrane was used as a scaffold (Figure 1E) for cell culture, and the liquid produced on pressing the CGF gel into the membrane was collected as CGF fluid (Figure 1D). 


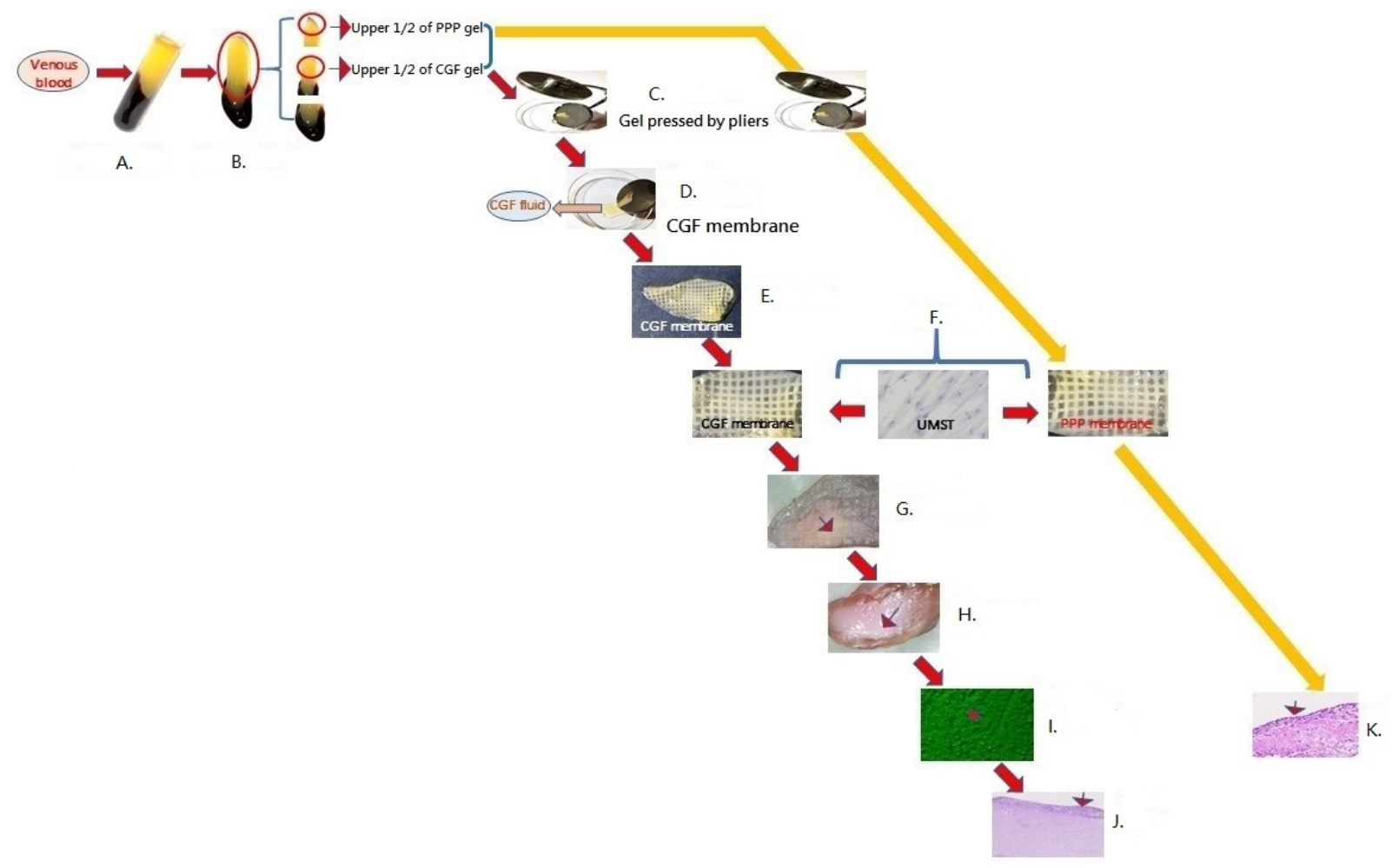

Figure 1. Flow chart showing the preparation of concentrated growth factors (CGF) or platelet-poor plasma (PPP) gel and membrane from venous blood. The CGF membrane and PPP membrane used as scaffolds for culturing umbilical cord mesenchymal stem cells in vitro. (A): CGF gel formed after fractional gradient centrifugation of venous blood. (B): The upper half of the PPP gel used for the preparation of PPP membrane. The upper half of the CGF gel used for the preparation of the CGF membrane and fluid. (C): the upper half of the CGF gel compressed by pliers. (D): after compression, CGF gel separated into the CGF membrane and CGF fluid. (E): CGF membrane used as a scaffold for cell culture. (F): Umbilical cord mesenchymal stem cells seeded onto the CGF membrane or PPP membrane. The above 2 kinds of membrane used as scaffolds for culturing umbilical cord mesenchymal stem cells in vitro. (G): photograph of umbilical cord mesenchymal stem cells and CGF membrane cultured for 2 weeks $(-)$. $(\mathbf{H})$ : photograph of umbilical cord mesenchymal stem cells and CGF membrane cultured for 4 weeks $({ })$. (I): at the end of the second culture stage, the cells proliferated on the roof of CGF membrane and appeared as cuboid shape cells $(\vec{V})$ observed in inverted microscope. (J): Multi-layered epidermal-like cells in the roof of a tissue section of umbilical cord mesenchymal stem cells and CGF membrane $(\boldsymbol{H})$ cultured for 4 weeks. Hematoxylin-eosin staining. (K): at the end of the second culture stage, multiple spindle-shaped cells distributed in the whole tissue sections $(\overrightarrow{ })$. Abbreviations: CGF—concentrated growth factors; H\&E staining-Hematoxylin-eosin staining; PPP_platelet-poor plasma; and UMST—umbilical cord mesenchymal stem cells.

\subsection{Culture of Human Umbilical Cord Wharton's Jelly Mesenchymal Stem Cell Line and HaCaT Cell Line}

The human umbilical cord Wharton's jelly mesenchymal stem cell (UMST) line (BCRC, WJMSC, RM60596) was purchased from the Bioresource Collection and Research Centre, Hsinchu, Taiwan. The culture medium used was Dulbecco's modified Eagle medium (DMEM/high glucose, cat no. SH30022.02, HyClone Industries, South Logan, UT, USA) plus $10 \%$ fetal bovine serum (FBS) (standard fetal bovine serum, cat no. SH30088.03, HyClone Industries, South Logan, UT, USA), $100 \mathrm{IU} / \mathrm{mL}$ penicillin and $100 \mu \mathrm{g} / \mathrm{mL}$ streptomycin (penicillin-streptomycin solution, cat no. 1220, HyClone Industries, South Logan, UT, USA). No additional epidermal growth factor was present in the medium, which was changed every 2-3 days. The cells were cultured in $5 \% \mathrm{CO} 2$ at a temperature of $37{ }^{\circ} \mathrm{C}$ in a humidified atmosphere. 
The HaCaT cell line provided by the Department of Dermatology of Kaohsiung Medical College were cultured on a CGF membrane, as described above. The medium used in the culture was Dulbecco's modified Eagle medium/low Glucose (HyClone SH30021.01), 10\% fetal bovine serum (HyClone, SH30088.03), $100 \mathrm{IU} / \mathrm{mL}$ penicillin and $100 \mu \mathrm{g} / \mathrm{mL}$ streptomycin (HyClone cat no.1220). The cells were cultured in 5\% CO2 at a temperature of $37^{\circ} \mathrm{C}$ in a humidified atmosphere, and the medium was changed every $2-3$ days. The $\mathrm{HaCaT}$ cell is a keratinocyte cell line, which can be a tool for comparisons with keratinocytes differentiated from umbilical cord mesenchymal stem cells.

\subsection{In Vitro Culture of Human Umbilical Cord Wharton's Jelly Mesenchymal Stem Cells or HaCaT Cells on CGF Membrane}

We added $1 \mathrm{~mL}$ of UMST suspension $\left(2 \times 10^{4}\right.$ cells $/ \mathrm{mL}$ ) (Figure $\left.1 \mathrm{~F}\right)$ to cover each CGF membrane $(3 \mathrm{~mm} \times 5 \mathrm{~mm})$ placed on a $35-\mathrm{mm}$ petri dish, and cultured UMST onto the CGF membrane using DMEM $+10 \%$ FBS as a medium. The tissue culturing process was divided into two stages, as follows: In the first stage, the air-fluid surface of the medium was at the uppermost portion of the CGF membrane (i.e., the medium covered the top of the CGF membrane) and was cultured for 2 weeks (Figure 1G). In the second stage, the air-fluid surface of the culture was constantly maintained beneath the uppermost portion of the CGF membrane (i.e., the surface of the CGF membrane was above the surface of the medium) for another 2 weeks (Figure $1 \mathrm{H}$ ). At the end of the second culture stage, the cells proliferated on the roof of CGF membrane and appeared as cuboid shape cells observed in an inverted microscope (Figure 1I). The cultured CGF membrane-UMST samples were used for sectioning tissues and histological staining (Figure 1J).

The steps for culturing HaCaT cell on CGF membrane were the same as the above steps for umbilical cord stem cells culture on CGF membrane.

\subsection{In Vitro Culture of Human Umbilical Cord Wharton's Jelly Mesenchymal Stem Cells on PPP Membrane}

We added $1 \mathrm{~mL}$ of UMST suspension $\left(2 \times 10^{4}\right.$ cells $\left./ \mathrm{mL}\right)$ (Figure $\left.1 \mathrm{~F}\right)$ to cover each PPP membrane $(3 \mathrm{~mm} \times 5 \mathrm{~mm})$ placed on a $35-\mathrm{mm}$ petri dish, and cultured UMST onto the PPP membrane using DMEM $+10 \%$ FBS as a medium. The tissue culturing process was divided into two stages, as follows: In the first stage, the air-fluid surface of the medium was at the uppermost portion of the PPP membrane (i.e., the medium covered the top of the PPP membrane) and was cultured for 2 weeks. In the second stage, the air-fluid surface of the culture was constantly maintained beneath the uppermost portion of the PPP membrane (i.e., the surface of the PPP membrane was above the surface of the medium) for another 2 weeks. The cultured PPP membrane-UMST samples were used for sectioning tissues and histological staining (Figure 1K).

\subsection{Histological Analysis}

For histological analysis, tissue specimens, including the UMST-CGF membrane, UMST-PPP membrane, and HaCaT cell-CGF membrane, were first fixed with $10 \%$ buffered formalin for $24 \mathrm{~h}$, embedded in paraffin and sectioned to a thickness of $3 \mu \mathrm{m}$. The tissue sections were subjected to hematoxylin-eosin (H\&E) staining, Masson trichome staining, and immunohistochemical staining [19].

\subsection{Immunohistochemical Analysis}

For immunohistochemical analysis, the preparation of tissue samples was the same as the steps mentioned above in the histological evaluation section [20]. Immunohistochemical staining was performed using the Ultra Vision Quanto Detection System HRP DAB (TL-060-QHD, Thermo Fisher Scientific Inc., Waltham, MA, USA). Antibodies included pancytokeratin antibody (PCK-26, GTX26401, GeneTex, Hsinchu, China), P63 antibody (GT1179, GTX633818, Hsinchu, China), and vimentin antibody (GT7812, GTX629743, GeneTex, Hsinchu, China). Pancytokeratin antibody, P63 antibody, and vimentin antibody were not added to the blank controls in immunohistochemical experiments. By immunohisto- 
chemical staining, we compared the expression of pancytokeratin, P63, and vimentin cell markers of (1) HaCaT cells or UMST after cultured on CGF membrane, and (2) UMST after cultured on PPP membrane.

\subsection{Digital Slide Scanning Analysis}

Of the total 6 sets of specimens in 3 groups of UMST-CGF membrane, UMST-PPP membrane, and $\mathrm{HaCaT}$ cells-CGF membrane, three tissue sections were cut from the tissue block of each specimen, placed on the same glass slide and measured separately for the following: (1) The maximum thickness of the multi-layer epidermal-like tissue formed on the upper surface of the concentrated growth factors membrane. The reason for choosing the maximum thickness of the epidermal-like tissue is that during the production of tissue sections and the staining process, the epidermal-like tissue on the tissue sections was easily separated from the fibrin clot and fell off, reducing the measured thickness. Thus, the maximum value in the tissue section was chosen; and (2) after immunohistochemical staining of the tissue sections of these tissues, a total of 100 cells were randomly counted in each field of vision to be observed, and the average number of the cells in the field of vision was calculated as number of positive cells expressing pancytokeratin marker, P63 marker, or vimentin marker in the tissue sections.

We used the Motic Digital Slide Scanner system (MoticEasyScan, Richmond, ON, Canada) for digital slide scanning analysis.

Statistical Evaluation

Student's $t$-test was used for statistical evaluation. A $p$ value $<0.05$ was considered significant.

\section{Results}

The in vitro tissue culture showed that human Wharton's jelly umbilical cord mesenchymal stem cells could attach to the concentrated growth factors membrane and proliferate.

At the end of the second culture stage, the cells proliferated on the roof of the CGF membrane and appeared as cuboid shape cells $(\vec{b})$ observed in an inverted microscope (Figure 1I), and a multi-layer epidermal-like tissue $(\lambda)$ formed on the surface of the concentrated growth factors membrane (Figure 1J). The thickness of the tissue ranged from $89.91 \mu \mathrm{m}$ to $204.19 \mu \mathrm{m}$ (mean $\pm \mathrm{SD}=144.36 \mu \mathrm{m} \pm 43.14 \mu \mathrm{m}$ ) (Figure $2 \mathrm{~A}$ ). We performed immunohistochemical staining on the sections of these multi-layer epidermallike tissues and found that $79.8 \% \pm 7.2 \%$ of the cells expressed the pancytokeratin marker (Figure 2B), 29.5\% $\pm 9.4 \%$ of the cells expressed the P63 marker (Figure 2C), and $71.7 \% \pm 3.9 \%$ of the cells expressed the vimentin marker (Figure 2D). In comparison, no labelled cells were found in the blank controls where a pancytokeratin, P63, or vimentin antibody was not added (Figure 2E). Under Masson trichome staining, thick dark-red fiber bands could be seen around the cell clusters in the tissue section. However, in other places where there was no cell clusters in the blood clot, pink was displayed (Figure 2F). 
UMST and CGF membrane

Cultured for 4 weeks

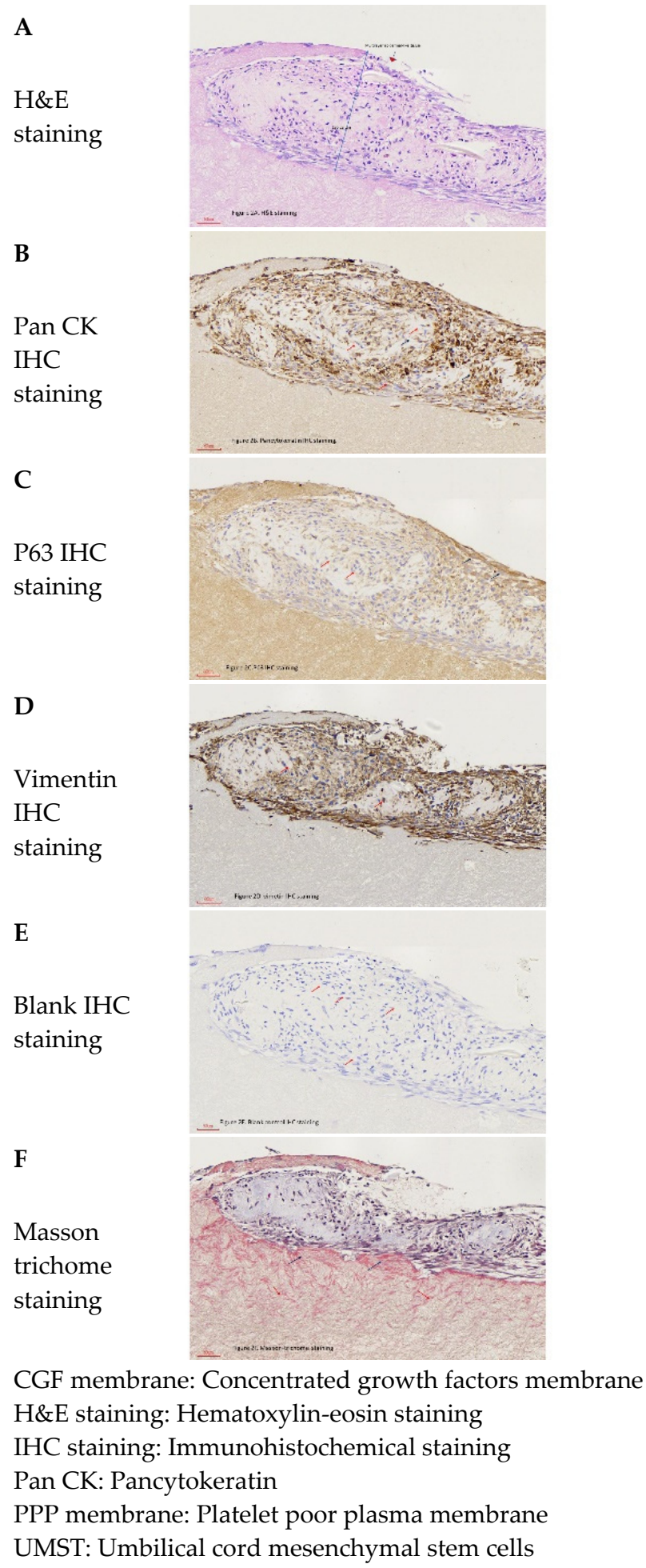

Figure 2. Human Wharton's jelly umbilical cord mesenchymal stem cells were implanted on the concentrated growth factors membrane and cultured for four weeks. Multi-layer epidermal-like tissue was formed on the surface of the concentrated growth factors membrane. Tissue sections were prepared from these tissue samples from the in vitro culture preparation. Hematoxylin-eosin staining, pancytokeratin and P63 cell marker immunohistochemical staining were performed. The results were compared with 
those from blank controls. (A) After four weeks of in vitro culture, multi-layer epidermal-like tissue $(\vec{D})$ formed on the surface of the concentrated growth factors membrane. The maximal thickness was $144.36 \mu \mathrm{m} \pm 43.14 \mu \mathrm{m}$ $($ mean $\pm \mathrm{SD})\left({ }^{\top}\right)$. (B) The tissue sections of multi-layer epidermal-like tissue further stained for pancytokeratin. Most cells were positive for pancytokeratin marker $(\boldsymbol{\gamma})$, and only a few cells were negative $(\boldsymbol{\Gamma})$. (C) The tissue sections of multi-layer epidermal-like tissue further stained for P63. Some cells were P63-positive ( $\boldsymbol{\gamma}$ ), while most cells were negative ( $\boldsymbol{T}$ ). (D) The tissue sections of multi-layer epidermal-like tissue further stained for vimentin. A few cells were vimentin-positive $(\boldsymbol{\gamma})$, while few cells were negative $(\boldsymbol{\gamma})$. (E) The blank controls of the multi-layer epidermal-like tissue were used for immunohistochemical staining. We found that the cell were pancytokeratin-negative, P63-negative, and vimentin-negative $(\boldsymbol{T})$. (F) under Masson trichome stain, thick dark-red fiber bands could be seen around the cell clusters in the tissue section ( $)$, while other fibrin clots without cell clusters were pink $(\bar{\gamma})$.

The in vitro tissue culture also showed that $\mathrm{HaCaT}$ cells could attach to the concentrated growth factors membrane and proliferate to form a multi-layer tissue (Figure 3A). The thickness of tissue ranged from $100.26 \mu \mathrm{m}$ to $61.17 \mu \mathrm{m}$ (mean $\pm \mathrm{SD}=74.05 \mu \mathrm{m} \pm 13.44 \mu \mathrm{m}$ ). We performed immunohistochemical staining on the sections of these multi-layer tissues and found that $88.1 \% \pm 4.9 \%$ of the cells expressed the pancytokeratin marker (Figure $3 \mathrm{~B}$ ), $63.6 \% \pm 11.4 \%$ of the cells expressed the P63 marker (Figure 3C), and 79\% $\pm 9.9 \%$ of the cells expressed the vimentin marker (Figure 3D). In comparison, no labelled cells were found in the blank control where a pancytokeratin, P63, or vimentin antibody was not added (Figure 3E). Under Masson trichome staining, thick blue fiber bands can be seen around the HaCaT cell clusters in the tissue section. However, in other places where there are no cell clusters in the blood clot, red is displayed (Figure 3F). The maximal thickness of the blue band in the tissue sections of each sample ranged from $169.92 \mu \mathrm{m}$ to $41.11 \mu \mathrm{m}$ (mean $\pm \mathrm{SD}=96.48 \mu \mathrm{m} \pm 44.7 \mu \mathrm{m}$ ).

The in vitro tissue culture showed that human Wharton's jelly umbilical cord mesenchymal stem cells could attach to the platelet-poor plasma (PPP) membrane and proliferate. At the end of the second culture stage, the cells proliferated and distributed in the whole-tissue section, and appeared as spindle shape cells (Figure 4A). We performed immunohistochemical staining on the tissue sections and found that $9.7 \% \pm 2.4 \%$ of the cells expressed the pancytokeratin marker (Figure $4 \mathrm{~B}$ ), $7.45 \% \pm 1.9 \%$ of the cells expressed the P63 marker (Figure 4C), and $95.9 \% \pm 3.7 \%$ of the cells expressed the vimentin marker (Figure 4D). In comparison, no labelled cells were found in the blank controls where a pancytokeratin, P3, or vimentin antibody was not added (Figure 4E). There were multiple thin blue bands in the tissue sections under Masson trichrome staining (Figure 4F).

Under Masson trichrome staining, there were significant changes between the tissues cultured by the UMST-CGF membrane, UMST-PPP membrane and HaCaT cell-CGF membrane. In the fibrin clot near the cell clusters, the tissue section of HaCaT cell-CGF membrane showed a blue thick band (Figure 3F), the tissue section of the UMST-CGF membrane showed a small amount of thicker red fibers (Figure 2F), and the tissue section of the UMST-PPP membrane showed a few thin blue fibers.

The results showing the percentage of cells expressing pancytokeratin, P63, and vimentin cell markers are summarized in Table 1 . The data were compared by paired t-test. A $p$ value $<0.05$ was considered significant (Table 1). In terms of the percentage of UMST expressing pancytokeratin, P63, and vimentin cells markers, there was significant difference between the UMST-CGF membrane and the UMST-PPP membrane groups (Table 1, comparison group 1). In terms of the percentage of UMST or HaCaT cells expressing pancytokeratin, P63, and vimentin cell markers, there was no significant difference between the UMST-CGF membrane and the HaCaT cell-CGF membrane groups (Table 1, comparison group 2). 


\section{HaCaT cells and CGF membrane}

Cultured for 4 weeks

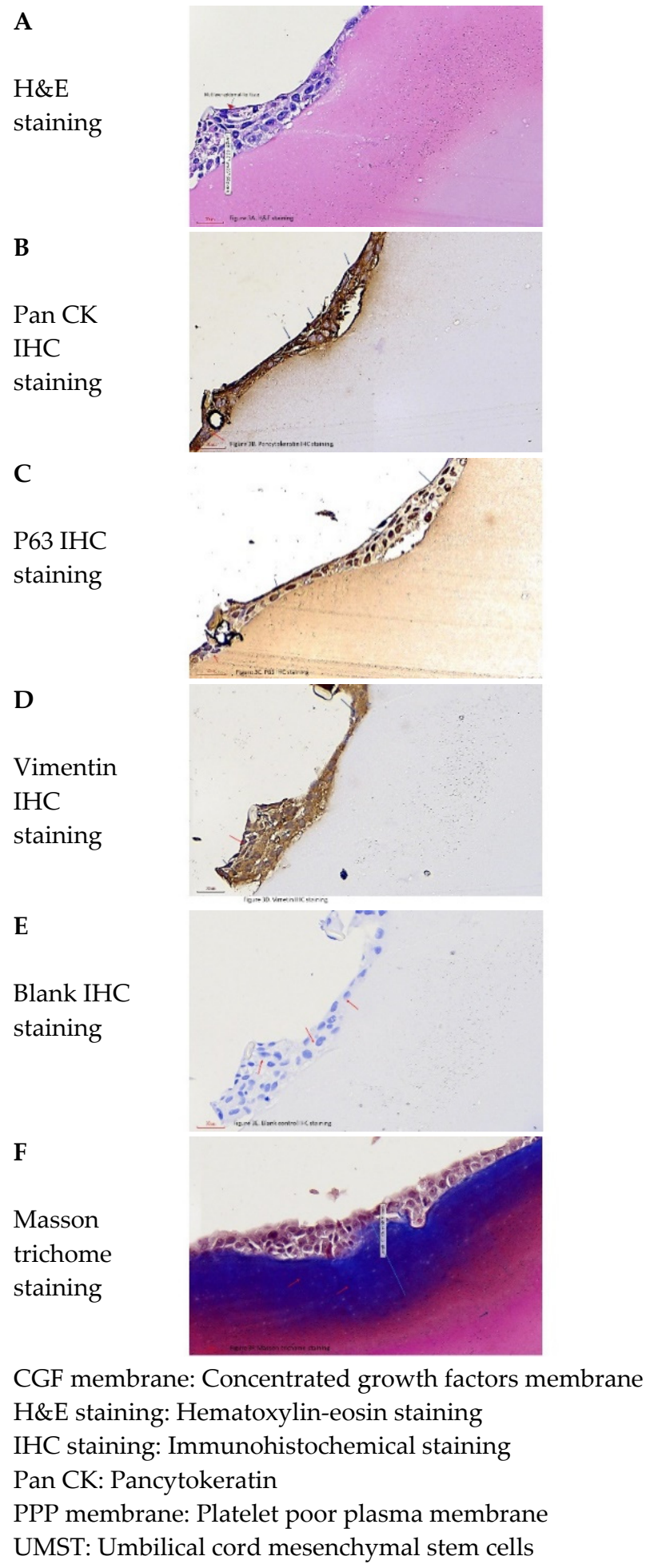

Figure 3. HaCaT cells were implanted on the concentrated growth factors membrane and cultured for four weeks. Multi-layer tissue was formed on the surface of the concentrated growth factors membrane. Tissue sections were prepared from these tissue samples from the in vitro culture preparation. Hematoxylin-eosin staining, pancytokeratin and P63 cell marker immunohistochemical staining were performed. The results were compared with those from blank controls. (A) After four 
weeks of in vitro culture, multi-layer tissue $(\vec{H})$ formed on the surface of the concentrated growth factors membrane. The maximal thickness was $74.05 \mu \mathrm{m} \pm 13.44 \mu \mathrm{m}$ (mean $\pm \mathrm{SD})(\nabla)$ ). (B) The tissue sections of multi-layer tissue further stained for pancytokeratin. Most cells were positive for pancytokeratin marker $(\boldsymbol{\lambda})$, and only a few cells were negative $(\boldsymbol{\nabla})$. (C) The tissue sections of multi-layer tissue further stained for P63.

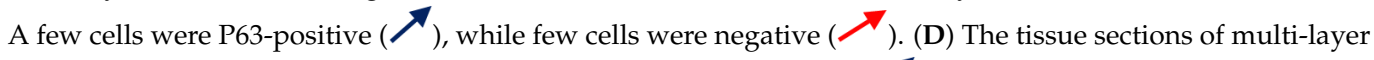
tissue further stained for vimentin. A few cells were vimentin-positive $(\boldsymbol{})$, while few cells were negative $(\boldsymbol{})$. (E) The blank controls of the multi-layer epidermal-like tissue were used for immunohistochemical staining. We found that the cell were pancytokeratin-negative, P63-negative, and vimentin-negative $(\boldsymbol{\nabla})$. (F) Under Masson trichome satin, thick blue fiber bands could be seen around the cell clusters in the tissue section $(\boldsymbol{\Gamma})$, while other fibrin clots without cell clusters were red $(\boldsymbol{\nearrow})$. The maximal thickness of the blue band in tissue sections of each sample ranged from $169.92 \mu \mathrm{m}$ to $41.11 \mu \mathrm{m}$ (mean $\pm \mathrm{SD}=96.48 \mu \mathrm{m} \pm 44.7 \mu \mathrm{m}$ ). Abbreviations: H\&E staining-hematoxylin-eosin staining; and IHC—immunohistochemical staining.

Table 1. Statitical analysis of percentage of cells expressing pancytokeratin, P-63, or vimentin marker in tissue formed by culturing UMST or HaCaT cells on CGF membrane, or UMST on PPP membrane.

\begin{tabular}{|c|c|c|c|c|}
\hline \multicolumn{5}{|c|}{ Comparsion group 1 percentage of cells expressing marker (Data= Mean \pm SD, N: Number of samples) } \\
\hline cell marker & $\begin{array}{c}\text { UMST + CGF } \\
\text { membrane group }\end{array}$ & $\begin{array}{c}\text { USMT + PPP } \\
\text { membrane group }\end{array}$ & t value & $p$ value \\
\hline pancytokeratin & $79.8 \% \pm 7.2 \%, \mathrm{~N}=6$ & $9.7 \% \pm 2.4 \%$ & 12.3 & $<0.05$ \\
\hline p-63 & $29.5 \% \pm 9.4 \%, \mathrm{~N}=6$ & $7.45 \% \pm 1.9 \%$ & 6.78 & $<0.05$ \\
\hline vimentin & $71.7 \% \pm 3.9 \%, \mathrm{~N}=6$ & $95.9 \% \pm 3.7 \%$ & 6.4 & $<0.05$ \\
\hline \multicolumn{5}{|c|}{ Comparsion group 2 percentage of cells expressing marker (Data $=$ Mean $\pm \mathrm{SD}, \mathrm{N}$ : Number of samples) } \\
\hline cell marker & $\begin{array}{c}\text { UMST + CGF } \\
\text { membrane group }\end{array}$ & $\begin{array}{l}\text { HaCaT cells + CGF } \\
\text { membrane group }\end{array}$ & t value & $p$ value \\
\hline pancytokeratin & $79.8 \% \pm 7.2 \%, \mathrm{~N}=6$ & $88.1 \% \pm 4.9 \%, \mathrm{~N}=6$ & 0.35 & $>0.05$ \\
\hline$p-63$ & $29.5 \% \pm 9.4 \%, \mathrm{~N}=6$ & $63.6 \% \pm 11.4 \%, \mathrm{~N}=6$ & 1.19 & $>0.05$ \\
\hline vimentin & $71.7 \% \pm 3.9 \%, \mathrm{~N}=6$ & $79 \% \pm 9.9 \%, \mathrm{~N}=6$ & 0.17 & $>0.05$ \\
\hline
\end{tabular}

CGF membrane: concentrated growth factors membrane. PPP membrane: platelet poor plasma membrane. UMST: umbilical cord mesenchymal stem cells. 


\section{UMST and PPP membrane}

Cultured for 4 weeks

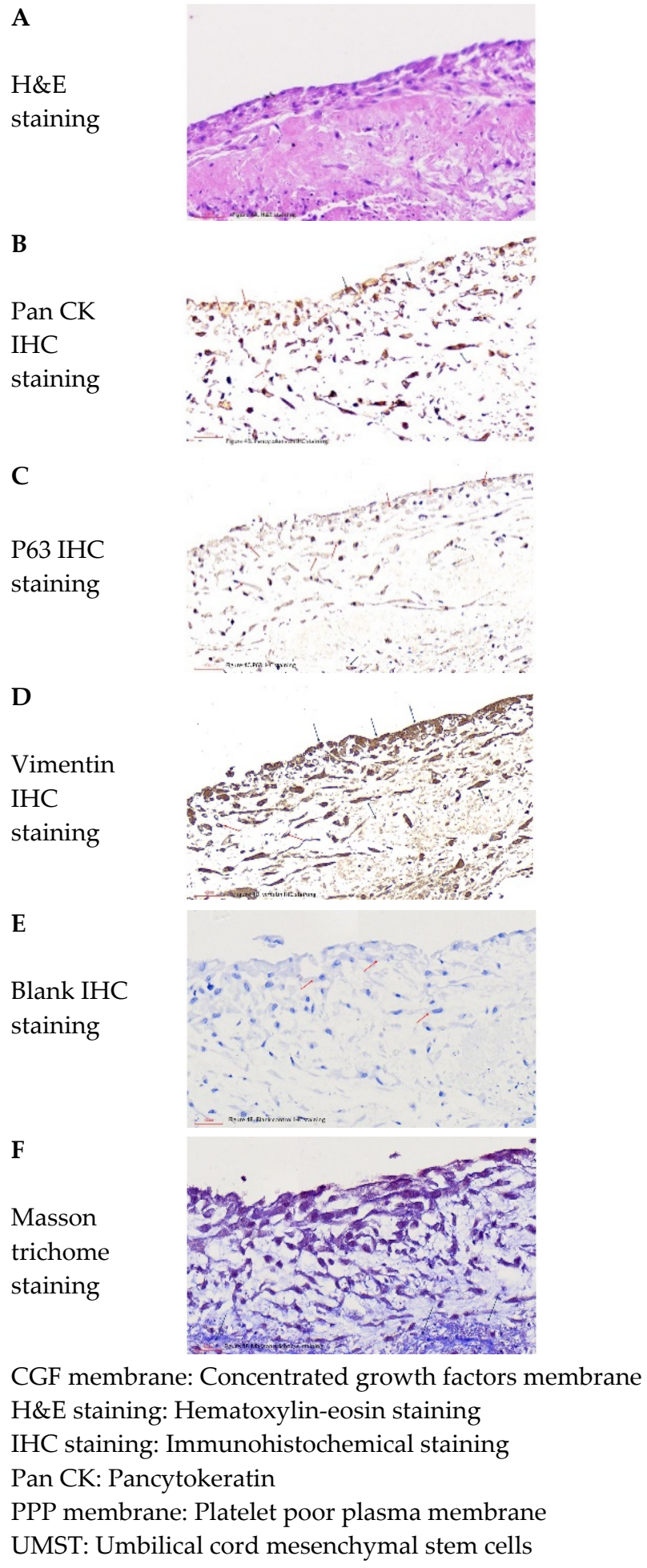

Figure 4. Human Wharton's jelly umbilical cord mesenchymal stem cells were implanted on the platelet-poor plasma membrane and cultured for four weeks. Tissue sections were prepared from these tissue samples from the in vitro culture preparation. Hematoxylin-eosin staining, pancytokeratin and P63 cell marker immunohistochemical staining were performed. The results were compared with those from blank controls. (A) after four weeks of in vitro culture, multiple spindle shaped cells 
) were distributed in whole-tissue sections of platelet-poor plasma membrane. (B) The tissue sections further stained for pancytokeratin. Only few cells were positive for pancytokeratin marker $(\nearrow)$, and most cells were negative $(\boldsymbol{\Gamma})$. (C) The tissue sections further stained for P63. Few cells were P63-positive $(\boldsymbol{\nearrow})$, while most cells were negative $\left(\boldsymbol{J}_{\text {). }}(\mathrm{D})\right.$ The tissue sections further stained for vimentin. Most cells were vimentin-positive $(\boldsymbol{\nearrow})$, while only few cells were negative $(\boldsymbol{J}$ ). (E) The blank controls of the multi-layer epidermal-like tissue were used for immunohistochemical staining. We found that the cells were pancytokeratin-negative, P63-negative, and vimentin-negative $(\boldsymbol{\nabla})$. (F) Under Masson trichome stain, multiple thin blue fiber bands could be seen around the cell clusters in the tissue section $(\boldsymbol{J})$. Abbreviations: H\&E staining-hematoxylin-eosin staining; and IHC staining-immunohistochemical staining.

\section{Discussion}

The results of this study showed that CGF membrane or PPP membrane could be used as a scaffold for UMST to attach and proliferate. However, in terms of the percentage of cells in the tissue sections expressing pancytokeratin, P63, and vimentin cell markers, there was a significant difference (Table 1). In other words, the CGF membrane could obviously promote UMST differentiation towards pancytokeratin and P63-expressing cells, but the PPP membrane cannot have this effect.

The results of this study show that the CGF membrane could be used as a scaffold not only for UMST but also for HaCaT cells to attach, proliferate, and form a multi-layer tissue. The above two cell populations composed of this tissue exhibit vimentin marker, pancytokeratin marker, and P63 marker, respectively. However, there was no statistically significant difference in the percentage of cells expressing the three different cell markers of the above two cell groups (Table 1).

All the above results tended to support the inference that part of the UMST cultivating in the CGF membrane differentiate towards keratinocyte-like cells (expressing only the pancytokeratin marker) and keratinocyte stem cell-like cells (expressing both pancytokeratin and P63 markers).

Previous studies reported that umbilical cord mesenchymal stem cells can be used for corneal epithelium regeneration [19] and differentiation into skin cells [16,17,21], thus playing a vital role in epithelial regeneration. However, the scaffold (such as the fibrin-agarose stroma substitute [22] or decellularized amniotic membrane [23]) and growth factors (50 $\mathrm{ng} / \mathrm{mL}$ recombinant epidermal growth factor) used in the mesenchymal stem cell culture were not derived from the body's own tissue in these studies. Also, previous literature stated that concentrated growth factors membranes prepared form autologous blood could be used as scaffolds for the attachment and proliferation of keratinocyte cells [10]. In this study, we found that the concentrated growth factors membranes prepared form autologous blood could not only serve as a scaffold for the attachment and proliferation of umbilical cord Wharton's jelly mesenchymal stem cells, but also provide an effective and autologous bioscaffold to differentiate mesenchymal stem cells towards keratinocyte stem cells, keratinocytes, and promote these cells to stack into a multi-layer epithelial-like tissue. This finding of the mesenchymal-epithelial transition can be attributed to the following factors:

(1) The 3D fibrin mesh in the concentrated growth factors membrane is suitable for the attachment and growth of mesenchymal stem cells;

(2) The concentrated growth factors membrane contains various growth factors and cytokines [4-6]. Using the same Medifuge centrifugation system (Silfradent, S.R,L, Santa Sofia, Italy) as ours, the growth factors and proinflammatory cytokines contents in concentrated growth factors (CGF) had been reported in detail [6]. It was reported that growth factors (such as epidermal growth factors, fibroblast growth factors, insulin-like growth factors, and vascular endothelial growth factor), and cytokine (such as interleukin 1, 4, and 6) might play important roles in tissue regeneration and wound healing [24]. It was also reported that some cytokines could extensively affect mesenchymal stem cell function $[25,26]$; 
(3) Therefore, it is proposed that the related growth factors and cytokines contained in CGF membrane play an important role in promoting mesenchymal stem cells differentiating toward keratinocytes;

(4) It can maintain the roof surface of the concentrated growth factors membrane where mesenchymal stem cells attach and grow on the air-fluid surface. When cultured in vitro for 2 weeks, the physical environment change can be used to promote the proliferation and overlap of keratinocyte-like cells into a multi-layer epidermal-like tissue;

(5) Under Masson trichome staining, the tissue section of the UMST-CGF membrane showed a small amount of thicker red fibers in the fibrin clot near the cell clusters (Figure 2F). It is speculated that mesenchymal stem cells and fibroblast are the same, and fibroblasts are in fact aged mesenchymal stem cells [27]. After attaching to the concentrated growth factors membrane, the mesenchymal stem cells may not only proliferate to form cell clusters, but also secrete extracellular matrix-like or collagenlike substances, which then fill on the original fibrin mesh to form a new dermis-like structure [28]. This change may stop the degradation of the CGF gel and remodel it to make it more suitable for mesenchymal stem cells to proliferate, differentiate towards keratinocytes and form an epidermal-like tissue. However, the quality and quantity of these substances released by mesenchymal stem cells are still subject to further research in the future;

(6) In terms of application in regenerative medicine, compared with previous literature reports on the method of transforming mesenchymal stem cells into keratinocyte-like cells by culture, the CGF membrane is a fully autologous fibrin mesh, growth factors, and cytokines, rather than heterologous [23] or artificial [22]. Of course, even in the same healthy adult, the composition of the CGF membrane will be different under different physiological conditions. However, our own CGF membrane is unique and it can avoid the problem of external infection or rejection, while the materials (autologous venous blood) are easy to obtain, the production process is simple and the cost is low.

Further studies are needed due to the following reasons:

(1) To understand the role of concentrated growth factors on different stem cells, such as bone marrow stem cells, adipose stem cells, or hair follicle stem cells;

(2) To develop efficient qualitative and quantitative methods to measure various effective ingredients in concentrated growth factors [29], and further explore the effects of its various growth factors (such as epidermal growth factor, transforming growth factor $\beta$, fibroblast growth factor, vascular endothelial growth factors, platelet-derived growth factors, and insulin-like growth factor) and cytokines (such as interleukin 1, 2, 4, 6) on promoting the differentiation of mesenchymal stem cells towards keratinocytes. Whether the promotion of stem cell differentiation towards keratinocytes was a result of a single component or multiple components remains to be determined;

(3) To develop a method that effectively preserves a large amount of concentrated growth factors membrane for large-scale use, such as large-scale trauma or burn;

(4) Together with different types of autologous stem cells, we can perform in vitro tissue culture under different environments, providing different autologous tissue, or using the same type of autologous stem cells, but gradually changing to different in vitro tissue environments and gradually transforming the same type of autologous stem cells into tissues in which cell groups of different characteristics coexist.

We hope that ultimately (1) autologous stem cells can be collected from patients; (2) stem cell growth medium prepared from autologous blood replaces animal preparation such as fetal bovine serum or bovine pituitary extract with autologous serum or concentrated growth factors preparation; and (3) biodegradable scaffolds (such as concentrated growth factors membrane made of autologous plasma fibrin) can be used as powerful tools for assisting autologous regenerative therapy. 
Funding: This research received no external funding.

Institutional Review Board Statement: The study was conducted according to the guidelines of the Declaration of Helsinki, and approved by the Institutional Review Board (or Ethics Committee) of Yuan's General Hospital(protocol code 20200602B and in 2020/07/09 of approval).

Informed Consent Statement: Informed consent was obtained from all subjects involved in the study.

Data Availability Statement: Data available in a publicly accessible repository.

Acknowledgments: The author wishes to thank Hsin-Su Yu and Hamm-Ming Sheu for guidance, Cheng-Che Eric Lan for providing the HaCaT cell line. Thank you to the Yuan's General Hospital for their assistance in the medical resources, and Joanne KUO and Wei-Chi Lee for the valuable support in document processing and data organization.

Conflicts of Interest: The authors declare no conflict of interest.

\section{References}

1. Borie, E.; Oliví, D.G.; Orsi, I.A.; Garlet, K. Platelet-rich fibrin application in dentistry: A litera-ture review. Int. J. Clin. Exp. Med. 2015, 8, 7922-7929.

2. Bonazza, V.; Borsani, E.; Buffoli, B.; Castrezzati, S.; Rezzani, R.; Rodella, L.F. How the different material and shape of the blood collection tube influences the Concentrated Growth Factors production. Microsc. Res. Tech. 2016, 79, 1173-1178. [CrossRef]

3. Pirpir, C.; Yilmaz, O.; Candirli, C.; Balaban, E. Evaluation of effectiveness of concentrated growth factor on osseointegration. Int. J. Implant. Dent. 2017, 3, 1-6. [CrossRef]

4. Amable, P.R.; Carias, R.B.V.; Teixeira, M.V.T.; Pacheco Ítalo, D.C.; Amaral, R.J.F.C.D.; Granjeiro, J.M.; Borojevic, R. Platelet-rich plasma preparation for regenerative medicine: Optimization and quantification of cytokines and growth factors. Stem. Cell Res. Ther. 2013, 4, 67. [CrossRef]

5. Yu, M.; Wang, X.; Liu, Y.; Qiao, J. Cytokine release kinetics of concentrated growth factors in different scaffolds. Clin. Oral Investig. 2018, 23, 1663-1671. [CrossRef]

6. Masuki, H.; Okudera, T.; Watanebe, T.; Suzuki, M.; Nishiyama, K.; Okudera, H.; Nakata, K.; Uematsu, K.; Su, C.-Y.; Kawase, T. Growth factor and pro-inflammatory cytokine contents in platelet-rich plasma (PRP), plasma rich in growth factors (PRGF), advanced platelet-rich fibrin (A-PRF), and concentrated growth factors (CGF). Int. J. Implant. Dent. 2016, 2, 1-6. [CrossRef]

7. Verma, R.; Negi, G.; Kandwal, A.; Chandra, H.; Gaur, D.S.; Harsh, M. Effect of autologous PRP on wound healing in dental regenerative surgeries and its correlation with PDGF levels. Asian J. Transfus. Sci. 2019, 13, 47-53. [CrossRef]

8. Tukaram, K.; Shirsagar, J.; Rubine, S. Innovation in regeneration-Concentrated growth factor. Int. J. Appl. Dent. Sci. 2017, 3, 206-208.

9. Zhang, M.; Park, G.; Zhou, B.; Luo, D. Applications and efficacy of platelet-rich plasma in dermatology: A clinical review. J. Cosmet. Dermatol. 2018, 17, 660-665. [CrossRef] [PubMed]

10. Kao, C. Use of concentrate growth factors gel or membrane in chronic wound healing: Description of 18 cases. Int. Wound J. 2019, 17, 158-166. [CrossRef] [PubMed]

11. Serra, R.; Buffone, G.; Dominijanni, A.; Molinari, V. Application of platelet-rich gel to enhance healing of trans metatarsal amputations in diabetic dysvascular patients. Int. Wound J. 2013, 10, 612-615. [CrossRef] [PubMed]

12. Houdek, M.T.; Wyles, C.C.; Stalboerger, P.G.; Terzic, A.; Behfar, A.; Moran, S.L. Collagen and Fractionated Platelet-Rich Plasma Scaffold for Dermal Regeneration. Plast. Reconstr. Surg. 2016, 137, 1498-1506. [CrossRef] [PubMed]

13. Barbon, S.; Stocco, E.; Macchi, V.; Contran, M.; Grandi, F.; Borean, A.; Parnigotto, P.P.; Porzionato, A.; De Caro, R. Platelet-Rich Fibrin Scaffolds for Cartilage and Tendon Regenerative Medicine: From Bench to Bedside. Int. J. Mol. Sci. 2019, $20,1701$. [CrossRef]

14. Llames, S.; Del Rio, M.; Larcher, F.; García, E.; García, M.; Escámez, M.J.; Jorcano, J.L.; Holguín, P.; Meana, A. Human plasma as a dermal scaffold for the generation of a completely autologous bioengineered skin. Transplant 2004, 77, 350-355. [CrossRef] [PubMed]

15. Ahmed, T.A.; Dare, E.V.; Hincke, M. Fibrin: A versatile scaffold for tissue engineering applications. Tissue Eng. Part B Rev. 2008, 14, 199-215. [CrossRef]

16. Santos, J.F.D.; Borcari, N.R.; Araujo, M.D.S.; Numes, V.A. Mesenchymal stem cells differentiate into keratinocytes and express epidermal kallikrelins: Towards an in vitro model of human epidermis. J. Cell. Biochem. 2019, 120, 13141-13155.

17. Martin-Piedra, M.A.; Alfonso-Rodriguez, C.A.; Zapater, A.; Durand-Herrera, D. Effective use of mesenchymal stem cells in human skin substitutes generated by tissue engineering. Eur. Cell Mater. 2019, 37, 233-249. [CrossRef]

18. Garzón, I.; Alfonso-Rodríguez, C.; Martínez-Gómez, C.; Carriel, V.; Martin-Piedra, M.; Fernández-Valadés, R.; Sanchez-Quevedo, M.; Alaminos, M. Expression of epithelial markers by human umbilical cord stem cells. A topographical analysis. Placenta 2014, 35, 994-1000. [CrossRef]

19. Azmi, S.M.; Salih, M.; Abdelrazeg, S.; Roslan, F.F.; Mohamed, R.; Jie, T.J.; Shaharuddin, B. Human umbilical cord-mesenchymal stem cells: A promising strategy for corneal epithelial regeneration. Regen. Med. 2020, 15, 1381-1397. [CrossRef] [PubMed] 
20. Maia, F.R.; Lourenço, A.H.; Granja, P.L.; Gonçalves, R.M.; Barrias, C.C.; Maia, R. Effect of Cell Density on Mesenchymal Stem Cells Aggregation in RGD-Alginate 3D Matrices under Osteoinductive Conditions. Macromol. Biosci. 2014, 14, 759-771. [CrossRef] [PubMed]

21. Fatima, Q.; Choudhry, N.; Choudhery, M.S. Umbilical Cord Tissue Derived Mesenchymal Stem Cells Can Differentiate into Skin Cells. Open Life Sci. 2018, 13, 544-552. [CrossRef] [PubMed]

22. Garzon, I.; Martin-Piedra, M.A.; Alfonso-Rodriguez, C.; Gonzalez-Andrades, M. Generation of Biomimetic Human Artificial Cornea Model Using Wharton's Jelly Mesenchymal Stem Cells. Cornea 2014, 5517, 4073-4083. [CrossRef]

23. Salah, R.A.; Mohamed, I.K.; EI-Badin, N. Development of decellularized amniotic membrane as a bioscaffold for bone marrowderived mesenchymal stem cells: Ultrastructural study. J. Mol. Histol. 2018, 49, 289-301. [CrossRef]

24. Verma, U.P.; Yadav Rk, D.; Gupta, A. Platelet-rich Fibrin: A Paradigm in Periodontal Therapy-A Systemic Review. J. Int. Soc. Prev. Community Dent. 2017, 7, 227-233.

25. Selich, A.; Ha, T.C.H.; Morgan, M.; Falk, C.S. Cytokine Selection of MSC clones with Different Functionality. Stem. Cell Rep. 2019, 13, 262-273. [CrossRef] [PubMed]

26. Katagiri, W.; Sakaguchi, K.; Kawai, T.; Wakayama, Y.; Osugi, M.; Hibi, H. A defined mix of cytokines mimics conditioned medium from cultures of bone marrow-derived mesenchymal stem cells and elicits bone regeneration. Cell Prolif. 2017, 50, e12333. [CrossRef]

27. Soundararajam, M.; Kannan, S. Fibroblasts and mesenchymal stem cells: Two sides of the same coin? J. Cell Physiol. 2018, 233, 9099-9109. [CrossRef]

28. Assis-Ribas, T.; Fernanda-Forri, M.; Winnischofer, S.M.B.; Sogayar, M.C. Extracellular matrix dynamics during mesenchymal stem cell differentiation. Dev. Biol. 2018, 437, 63-74. [CrossRef]

29. Astori, G.; Arrati, E.; Bambi, F.; Benardi, M. Platelet lysate as a substitute for animal serum for the ex-vivo explanation of mesenchymal stem/stromal cells: Present and future. Stem. Cell Res. Ther. 2016, 7, 93. [CrossRef] [PubMed] 